\title{
Caracterização da família Geometridae (Insecta: Lepidoptera) associada a diferentes fragmentos florestais, em Cotriguaçu, MT
}

\author{
Aline Bispo Santos Januário ${ }^{1}$, Otávio Peres Filho ${ }^{1}$, Marcelo Dias de Souza ${ }^{2}$, Alberto Dorval' ${ }^{1}$, Marcelo Muniz Silva ${ }^{1}$ \\ ${ }^{1}$ Universidade Federal de Mato Grosso, Av. Fernando Corrêa da Costa, n 2367, Boa Esperança, CEP 78060-900, Cuiabá, MT, Brasil \\ Universidade Federal do Paraná, Rua Lothário Meissner, nº 900, CEP 80210-170, Jardim Botânico, Curitiba, PR, Brasil
}

"Autor correspondente:

aline-bis@bol.com.br

Termos para indexação:

Synchlora

Semiothisa

Entomofauna

Biodiversidade

\section{Index terms:}

Synchlora

Semiothisa

Entomofauna

Biodiversity

Histórico do artigo:

Recebido em 18/06/2012

Aprovado em 25/11/2013

Publicado em 31/12/2013

doi: 10.4336/2013.pfb.33.76.399
Resumo - Este trabalho teve como objetivo coletar, identificar e caracterizar faunisticamente a entomofauna de Geometridae no município de Cotriguaçu, MT, em diferentes fragmentos florestais. Foram amostrados os seguintes ambientes: plantio de Tectona grandis; plantio consorciado composto por espécies de Ficus spp., Astronium sp., Jacaranda copaia, Syzygium jambolanum e Aspidosperma sp., área de capoeira; mata ripária; e floresta nativa. O período de levantamento ocorreu no período de Julho de 2007 a Junho de 2008. Em cada ambiente foram utilizadas três armadilhas luminosas instaladas a 1,5 m do solo, modelo Luiz de Queiroz, ligadas quinzenalmente das 18:00 as 6:00 h. Foram determinados os índices faunísticos de dominância, frequência, abundância e constância. Foram coletados 387 indivíduos, divididos em 10 gêneros e 25 espécies. $\mathrm{O}$ ambiente com plantio consorciado foi o que apresentou o maior quantidade de indivíduos coletados. No entanto, a floresta nativa foi o ambiente que apresentou maior número de espécies coletadas e maior índice de diversidade. As espécies Semiothisa sp.3, Synchlora gerularia e Synchlora sp.2 foram dominantes em todos os ambientes estudados.

\section{Characterization of Geometridae family (Insecta: Lepidoptera) associated with different forest fragments in Cotriguaçu, Mato Grosso State, Brazil}

\begin{abstract}
The objective of this study was to collect, identify and carry out a faunistic characterization of Geometridae family in Cotriguaçu county, Mato Grosso State, Brazil in different forest fragments. The sampled sites were: Tectona grandis plantation; mixed stand formed by Ficus spp., Astronium sp., Copaia Jacaranda, Syzygium jambolanum and Aspidosperma sp., initial forest regeneration, riparian forest and native forest. The samples were collected from July 2007 to June 2008. In each site, three light traps were placed at $1.5 \mathrm{~m}$ above the soil. It was used the model Luiz of Queiroz, with lights twice a month from 6:00pm to 6:00am. The faunistic index of dominance, frequency, abundance and constancy were determined. It was collected 387 individuals, divided in 10 genus and 25 species. The mixed plantation presented the highest number of individuals collected. However, in the native forest it was observed the higher number of species and diversity. The species Semiothisa sp.3, Synchlora gerularia e Synchlora sp.2 were dominant in all studied sites.
\end{abstract}




\section{Introdução}

Com a crescente necessidade de novas áreas para fins econômicos, como agricultura e pecuária, ou simplesmente para urbanização, percebe-se cada vez mais a substituição ou a alteração dos ecossistemas. Desmatamentos e formação de pastagens levam a uma perda na riqueza e/ou diversidade de espécies de diversos grupos de insetos (Margules \& Pressy, 2000).

A grande diversidade de insetos em fragmentos de um mesmo ecossistema tem levado a investigações minuciosas das suas diferenças, principalmente, quando se trata de organismos que são sensíveis às pequenas variações ambientais, como os lepidópteros, que são considerados importantes indicadores da ação antrópica no ambiente (Brown Junior \& Freitas, 2000). Os lepidópteros constituem uma das principais ordens de insetos, com aproximadamente 146.000 espécies descritas. É um grupo que apresenta íntima associação com seu habitat e grande sensibilidade às suas mudanças, constituindo-se em indicadores da qualidade ambiental e integridade de paisagens naturais (Motta, 2002).

Em paisagens antrópicas muito fragmentadas, apenas as espécies de lepidópteros resistentes ou colonizadoras, de hábitos generalistas e adaptadass às condições desfavoráveis do meio, podem atingir densidades populacionais muito altas. Em geral, essas espécies invadem rapidamente estes habitats, modificando a diversidade local. Segundo Yamamoto \& Sota (2007), a família Geometridae é uma das mais diversificadas dentro da Ordem Lepidoptera, com mais de 21.000 espécies, tendo registro para diferentes regiões e ambientes, exceto para as regiões polares.

Os Lepidópteros podem se tornar pragas, refletindo em prejuízos econômicos em plantios florestais e na agricultura (Fonseca et al., 2006). Devido às intrigantes características biológicas desta família e o status de praga dado a algumas espécies desfolhadoras, o aspecto bioecológico das espécies de importância florestal tornou-se objeto de estudos desenvolvidos por Pratissoli \& Fonazier (1999), Santos et al. (2002) e Peres Filho et al. (2010).

As características populacionais de muitas espécies de lepidópteros ainda são desconhecidas, sendo importante monitorar a sazonalidade da população, para a correta indicação de alerta e possível controle. Segundo Nakano \& Leite (2000), a maior parte dos insetos-praga são fototrópicos positivo, sendo as mariposas um bom exemplo. Por esse motivo, as flutuações populacionais destas espécies podem ser monitoradas com o uso de armadilha luminosa adequada à coleta de insetos de hábitos noturnos ou crepusculares (Silveira Neto et al., 1976).

O objetivo deste trabalho foi identificar, quantificar e desenvolver estudos faunísticos e de similaridade com a entomofauna de geometrídeos coletadas nos diferentes ambientes florestais no Município de Cotriguaçu, estado de Mato Grosso, uma vez que esta família tem muitos insetos considerados pragas e que se alimentam de diversas espécies vegetais, o que torna importante seus estudos em ambientes florestais.

\section{Material e métodos}

O estudo foi realizado de julho de 2007 a junho de 2008, na fazenda São Nicolau, propriedade da Organización Nacionale du Fôret/Peugeot - ONF Brasil, localizada na região amazônica do noroeste do estado de Mato Grosso, no Município de Cotriguaçu. O clima na região, de acordo com a classificação de Köppen, pertence ao grupo A - Clima tropical chuvoso. O tipo climático é o "Am", que intercala pequeno período de seca e chuvas inferiores a $60 \mathrm{~mm}$ no mês mais seco. As temperaturas médias anuais são elevadas durante o ano, oscilando entre $23{ }^{\circ} \mathrm{C}$ e $25^{\circ} \mathrm{C}$, sendo os meses da primavera-verão, os mais quentes e com elevada precipitação pluviométrica (Projeto RADAM, 1982).

A área total da fazenda é de 10.134,43 ha, sendo $2.907,55$ ha $(28,68 \%)$ de área desmatada (floresta nativa que foi substituída por pastagem entre 1974 e 1997), $7.226,88$ ha $(71,31 \%)$ como área de floresta nativa remanescente, na qual $6.932,74$ ha $(68,40 \%)$ são constituídos de reserva legal da propriedade, da qual não podem ser utilizados para nenhuma finalidade. A área apresenta 2.000 ha $(19,73 \%)$ reservados para o reflorestamento de Tectona grandis e plantios consorciados de diferentes espécies, que vem sendo reflorestados ao longo dos anos, das quais foram amostrados os ambientes: 1) Plantio de T. grandis (teca); 2) Plantio consorciado composto por espécies de Ficus spp. (figueira), Astronium sp. (aroeira), Jacaranda copaia (caroba), Syzygium jambolanum (Jamelão) e Aspidosperma sp. (peroba); 3) Área de Capoeira; 4) Mata ripária; 5) Floresta nativa. 
Nas coletas dos espécimes de geometrídeos foram empregadas armadilhas luminosas semelhantes ao modelo "Luiz de Queiroz" (Silveira-Neto \& Silveira, 1969), adaptadas com lâmpadas ultravioleta fluorescente de 15 watts e 100 volts, sendo diferenciadas apenas pela colocação de uma sacola plástica (saco de lixo de 100 litros com papel picado, que dificulta a fuga dos lepidópteros) (Ferreira \& Martins, 1982). As armadilhas foram instaladas a $1,5 \mathrm{~m}$ do solo, em instrumento denominado "forca", (Figura 1) acionadas por baterias automotivas das $18 \mathrm{~h}$ às $6 \mathrm{~h}$.

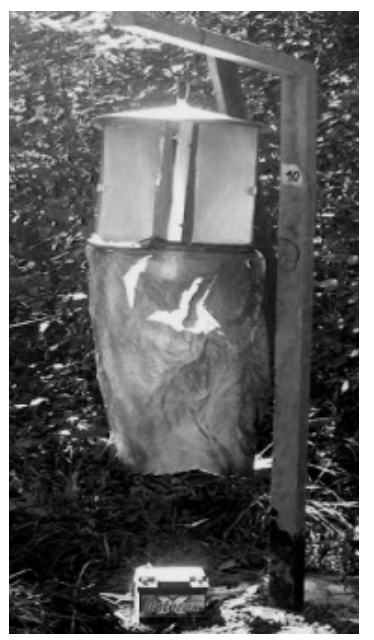

Figura 1. Armadilha luminosa pendurada no instrumento chamado "forca", utilizada nas coletas realizadas na fazenda São Nicolau, Cotriguaçu, MT, 2007-2008.
Foram distribuídas ao acaso três armadilhas por ambiente, entre as linhas do plantio, totalizando 15 armadilhas (Tabela 1). Foram realizadas 24 coletas com intervalos quinzenais que foram convertidas em valores mensais para efeito de análise. O material coletado foi acondicionado em sacos de plástico e etiquetado com a data e identificação para cada armadilha, sendo posteriormente enviado para o Laboratório de Proteção Florestal, da Faculdade de Engenharia Florestal da Universidade Federal de Mato Grosso (UFMT) e acondicionado em freezer.

Os espécimes coletados foram triados, quantificados, codificados e encaminhados para identificação junto ao Museu de Entomologia da Escola Superior de Agricultura "Luiz de Queiroz" (ESALQ/USP). Os insetos foram identificados ao nível de gênero e quando possível em espécie.

Foram realizados estudos faunísticos para definição das classes de dominância dos indivíduos em cada ambiente, da qual foi adotado o método de Sakagami \& Laroca (1967) e Laroca \& Mielke (1975), que expressam se o inseto se encontra adaptado ao ambiente. Foram relatados também a abundância, frequência e constância de cada inseto, sendo que a frequência expressa a ocorrência do individuo em cada coleta; a constância se há casualidade da ocorrência do indivíduo na coleta; e a abundância determina se o indivíduo ocorre abundantemente no ambiente. Para para essas análises foi utilizando o programa ANAFAU (Moraes et al., 2003).

Tabela 1. Características dos ambientes estudados nas coletas na fazenda São Nicolau, Cotriguaçu, MT, 2007-2008.

\begin{tabular}{|c|c|c|c|c|c|}
\hline Ambiente & Armadilha & $\begin{array}{c}\text { Cobertura } \\
\text { florestal }\end{array}$ & $\begin{array}{l}\text { Ano de } \\
\text { plantio }\end{array}$ & $\begin{array}{c}\text { Espaçamento do plantio } \\
\text { (m) }\end{array}$ & Principais espécies \\
\hline \multirow{3}{*}{1} & 1 & Florestamento & 2002 & $3 \times 2$ & Tectona grandis \\
\hline & 2 & Florestamento & 2002 & $3 \times 2$ & Tectona grandis \\
\hline & 3 & Florestamento & 2001 & $3 \times 3$ & Tectona grandis \\
\hline \multirow{3}{*}{2} & 4 & Florestamento & 2002 & $6 \times 3$ & 4 espécies de Ficus e Jacaranda copaia \\
\hline & 5 & Florestamento & 2002 & $6 \times 3$ & 3 espécies de Ficus e Jacaranda copaia \\
\hline & 6 & Florestamento & 2002 & $6 \times 3$ & $\begin{array}{l}2 \text { espécies de Ficus, Astronium sp., } \\
\text { Aspidosperma sp. e Syzygium jambolanum }\end{array}$ \\
\hline \multirow{3}{*}{3} & 7 & Mata ripária & --- & --- & --- \\
\hline & 8 & Mata ripária & --- & --- & --- \\
\hline & 9 & Mata ripária & --- & --- & --- \\
\hline \multirow{3}{*}{4} & 10 & Capoeira & --- & --- & --- \\
\hline & 11 & Capoeira & --- & --- & --- \\
\hline & 12 & Capoeira & --- & --- & --- \\
\hline \multirow{3}{*}{5} & 13 & Floresta nativa & --- & --- & --- \\
\hline & 14 & Floresta nativa & --- & --- & --- \\
\hline & 15 & Floresta nativa & --- & --- & --- \\
\hline
\end{tabular}


A diversidade foi calculada pela equação proposta por Margalef (1951), que demonstra o quanto os ambientes são diversificados. É usada para comparar os ambientes em relação à distribuição dos indivíduos que ocorrem no local, da qual se obtém pela a razão entre o número de espécies no ambiente menos um e o logaritmo neperiano (Ln) do número de indivíduos do ambiente estudado. Foi determinada a flutuação populacional das espécies descritas como dominantes em todos os ambientes analisados. Para verificar o grau de similaridade entre os ambientes, foi realizada análise de cluster através do método proposto por Ward (1963), com o uso do programa Statistic 7.

Para verificar a preferência significativa dos insetos dentro dos ambientes amostrados, o delineamento considerado foi inteiramente casualizado, em esquema fatorial (espécies x ambiente). Os dados foram submetidos à análise de variância (ANOVA) e as médias comparadas pelo teste de Scott-Knott ao nível 5\% de probabilidade de erro, levando-se em conta a distribuição dos valores de contagem dos geometrídeos coletados nos diferentes fragmentos florestais. Utilizou-se a transformação dos dados usando a formula: $\sqrt{X+0,5}$, para a uniformização e normalidade dos dados (Phillips, 1990; Dorval et al., 2004). As análises foram executadas através do software SISVAR (Ferreira, 2008).

\section{Resultados e discussão}

Foram coletados 387 indivíduos da família Geometridae nos cinco ambientes amostrados. No plantio consorciado, foram coletados 114 indivíduos, o que correspondeu a $29,5 \%$ do total (Tabela 2). A maior quantidade de indivíduos coletados neste ambiente pode ter sido influenciada pela maior disponibilidade de alimentos, uma vez que se trata de um plantio heterogêneo, com oferta de diferentes itens alimentares ao longo do ano, tais como diferentes folhas e néctar de flores, onde os insetos não necessitam de competição pelo alimento. Nos ambientes de floresta nativa, plantio de $T$. grandis, mata ripária e capoeira, foram coletados 93, 69, 60 e 51 indivíduos de geometrídeos, respectivamente.

Foram identificadas 25 espécies de 10 gêneros, sendo Semiothisa o gênero com maior número de indivíduos coletados e com uma espécie dominante (Semiothisa sp3.) em todos os ambientes estudados. A presença desse gênero já foi relatada em culturas agrícolas no Brasil.
Segundo Lourenção et al. (1980), com a expansão da área de cultivo da soja no Brasil, novos insetos têm sido acrescidos à literatura se alimentando da cultura, dentre eles a espécie Semiothisa abydata, que foi registrada em plantios de soja se alimentando de diferentes variedades.

Tabela 2. Quantidade de espécies e de indivíduos da família Geometridae coletados nos cinco ambientes florestais da fazenda São Nicolau, em Cotriguaçu, MT. 2007-2008.

\begin{tabular}{|c|c|c|c|c|c|}
\hline \multirow{2}{*}{ Espécie } & \multicolumn{5}{|c|}{ Ambientes* } \\
\hline & 1 & 2 & 3 & 4 & 5 \\
\hline $\begin{array}{l}\text { Epimecis puellaria } \\
\text { (Guen., 1857) }\end{array}$ & - & - & - & 1 & 2 \\
\hline Epimecis sp.1 & - & - & 1 & 3 & - \\
\hline Epimecis sp.2 & - & - & 3 & - & - \\
\hline $\begin{array}{l}\text { Leuciris minutepunctaria } \\
\text { (Oberthur, 1916) }\end{array}$ & - & - & - & 1 & - \\
\hline Oospila sp. & 3 & 1 & - & 2 & 7 \\
\hline $\begin{array}{l}\text { Oxydia agicata } \\
\text { (Guen., 1857) }\end{array}$ & - & - & - & - & 3 \\
\hline Oxydia vesulia (Cr., 1779) & - & - & 1 & - & - \\
\hline Oxydia sp. & - & - & 1 & 1 & 1 \\
\hline $\begin{array}{l}\text { Pero amanda } \\
\text { (Druce, 1898) }\end{array}$ & 1 & - & 1 & 1 & 3 \\
\hline Pero sp. 1 & 1 & 2 & - & - & 1 \\
\hline Pero sp.2 & - & 1 & - & - & 1 \\
\hline Pero sp. 3 & 1 & - & - & - & - \\
\hline Pero sp.4 & 4 & 6 & - & - & 2 \\
\hline Pero sp.5 & - & - & 1 & - & - \\
\hline Phrudocentra sp. & 1 & 4 & - & 3 & 5 \\
\hline Semiothisa sp.1 & 2 & 1 & - & - & 6 \\
\hline Semiothisa sp.2 & 11 & 21 & 4 & - & 5 \\
\hline Semiothisa sp. 3 & 32 & 56 & 25 & 19 & 29 \\
\hline $\begin{array}{l}\text { Sphacelodes vulneraria } \\
\text { (Hueb., 1825) }\end{array}$ & 1 & 1 & - & - & 1 \\
\hline $\begin{array}{l}\text { Synchlora gerularia } \\
\text { (Hueb., 1823) }\end{array}$ & 11 & 6 & 12 & 13 & 8 \\
\hline Synchlora sp.1 & - & 1 & - & 1 & 2 \\
\hline Synchlora sp.2 & 1 & 13 & 7 & 5 & 15 \\
\hline Synchlora sp.3 & - & - & - & - & 1 \\
\hline Synchlora sp.4 & - & - & - & - & 1 \\
\hline $\begin{array}{l}\text { Thyrinteina schadeana } \\
\text { (Schs., 1927) }\end{array}$ & - & 1 & 4 & 1 & - \\
\hline Total & 69 & 114 & 60 & 51 & 93 \\
\hline
\end{tabular}


No plantio de $T$. grandis e no plantio consorciado foram encontradas 12 e 13 espécies de geometrídeos, respectivamente, com oito espécies acidentais em cada ambiente. As espécies Semiothisa sp.2, Semiothisa sp.3, Synchlora gerularia e Synchlora sp.2 foram dominantes em ambos os ambientes. Por se tratarem de floresta plantada o índice de diversidade foi semelhante nos dois ambientes, 2,59 e 2,53 respectivamente (Tabela 3). Segundo Margalef (1972), esse índice raramente ultrapassa o valor de 4,5, variando normalmente entre 1,5 e 3,5 , onde valores baixos representa um resultado de maior dominância de alguns indivíduos em relação aos outros grupos taxonômicos encontrados no ambiente estudado.

Tabela 3. Análise faunística de geometrídeos coletados com armadilha luminosa no plantio de Tectona grandis (ambiente 1) e no plantio consorciado composto por espécies de Ficus spp., Astronium sp., Jacaranda copaia, Syzygium jambolanum e Aspidosperma sp (ambiente 2) na fazenda São Nicolau, em Cotriguaçu, MT, 2007 - 2008.

\begin{tabular}{|c|c|c|c|c|c|c|c|c|c|c|}
\hline \multirow{2}{*}{ Espécie } & \multicolumn{5}{|c|}{ Ambiente 1} & \multicolumn{5}{|c|}{ Ambiente 2} \\
\hline & $\mathbf{D}^{1}$ & $\mathbf{D}^{2}$ & $\mathbf{A}$ & $\mathbf{F}$ & $\mathbf{C}$ & $\mathbf{D}^{1}$ & $\mathbf{D}^{2}$ & $\mathbf{A}$ & $\mathbf{F}$ & C \\
\hline Oospila sp. & nd & nd & $\mathrm{c}$ & $\mathrm{f}$ & $\mathrm{z}$ & nd & nd & $\mathrm{d}$ & $\mathrm{pf}$ & $\mathrm{z}$ \\
\hline Pero amanda & nd & nd & $\mathrm{d}$ & $\mathrm{pf}$ & $\mathrm{z}$ & - & - & - & - & - \\
\hline Pero sp.1 & nd & nd & $\mathrm{d}$ & $\mathrm{pf}$ & $\mathrm{z}$ & nd & nd & $\mathrm{c}$ & $\mathrm{f}$ & $\mathrm{z}$ \\
\hline Pero sp.2 & - & - & - & - & - & nd & nd & $\mathrm{d}$ & $\mathrm{pf}$ & $\mathrm{z}$ \\
\hline Pero sp. 3 & nd & nd & $\mathrm{d}$ & $\mathrm{pf}$ & $\mathrm{z}$ & - & - & - & - & - \\
\hline Pero sp.4 & nd & $\mathrm{d}$ & $\mathrm{c}$ & $\mathrm{f}$ & $\mathrm{z}$ & $\mathrm{d}$ & $\mathrm{d}$ & $\mathrm{c}$ & $\mathrm{f}$ & $\mathrm{y}$ \\
\hline Phrudocentra sp. & nd & nd & $d$ & $\mathrm{pf}$ & $\mathrm{z}$ & nd & nd & $\mathrm{c}$ & $\mathrm{f}$ & $\mathrm{z}$ \\
\hline Semiothisa sp.1 & nd & nd & $\mathrm{c}$ & $\mathrm{f}$ & $\mathrm{z}$ & nd & nd & d & $\mathrm{pf}$ & $\mathrm{z}$ \\
\hline Semiothisa sp.2 & $\mathrm{sd}$ & $\mathrm{sd}$ & sa & sf & $\mathrm{w}$ & $\mathrm{d}$ & $\mathrm{d}$ & $\mathrm{ma}$ & $\mathrm{mf}$ & $\mathrm{y}$ \\
\hline Semiothisa sp.3 & $\mathrm{d}$ & $\mathrm{d}$ & $\mathrm{ma}$ & $\mathrm{mf}$ & $\mathrm{y}$ & $\mathrm{sd}$ & sd & sa & sf & $\mathrm{w}$ \\
\hline Sphacelodes vulneraria & nd & nd & $d$ & $\mathrm{pf}$ & $\mathrm{z}$ & nd & nd & d & $\mathrm{pf}$ & $\mathrm{z}$ \\
\hline Synchlora gerularia & $\mathrm{d}$ & $\mathrm{d}$ & $\mathrm{ma}$ & $\mathrm{mf}$ & $\mathrm{y}$ & $\mathrm{d}$ & d & $\mathrm{c}$ & $\mathrm{f}$ & $\mathrm{y}$ \\
\hline Synchlora sp.1 & - & - & - & - & - & nd & nd & d & $\mathrm{pf}$ & $\mathrm{z}$ \\
\hline Synchlora sp.2 & $\mathrm{d}$ & $\mathrm{d}$ & $\mathrm{ma}$ & $\mathrm{mf}$ & $\mathrm{y}$ & $\mathrm{d}$ & $\mathrm{d}$ & $\mathrm{ma}$ & $\mathrm{mf}$ & $\mathrm{w}$ \\
\hline Thyrinteina schadeana & - & - & - & - & - & nd & nd & $\mathrm{d}$ & $\mathrm{pf}$ & $\mathrm{z}$ \\
\hline Diversidade & & & 2,59 & & & & & 2,53 & & \\
\hline
\end{tabular}

D1 = dominância pelo método de Larroca e Mielke (1975); D2 = dominância pelo metodo de Sakagami e Larroca (1967) - (sd) super dominante, (d) dominante, (nd) não dominante; $\mathrm{A}$ = abundância - (sa) super abundante, (ma) muito abundante, (a) abundante, (c) comum, (d) dispersa, (r) rara; $\mathrm{F}=$ frequência - (mf) muito frequente, (f) frequente, (pf) pouco frequente; $\mathrm{C}=$ constância - (w) constante, (y) acessória, (z) acidental.

Na mata ripária e na capoeira, foram encontradas 11 e 12 espécies de geometrídeos, respectivamente, com 7 e 8 espécies acidentais e 4 e 3 acessórias, respectivamente. As espécies Semiothisa sp.3 e Synchlora gerularia foram dominantes, muito frequentes e abundantes nos dois ambientes. $\mathrm{O}$ índice de diversidade foi maior no ambiente capoeira $(2,79)$ em relação à mata ripária $(2,44)$ (Tabela 4$)$.

$\mathrm{Na}$ floresta nativa ocorreu a maior quantidade de espécies de geometrídeos, sendo dez espécies frequentes e duas constantes, das quais Oospila sp., Semiothisa sp.1, Semiothisa sp.3, Synchlora gerularia e Synchlora sp.2 foram dominantes e frequentes neste ambiente (Tabela 5). A maior diversidade foi observada na floresta nativa $(3,75)$, sendo considerado o ambiente mais diversificado, mais estável e conservado, com melhores condições de desenvolvimento e maior equilíbrio ambiental para esses insetos em relação aos outros ambientes estudados.

Embora a mata ripária assemelhe-se à floresta nativa em relação à vegetação, o fato do índice de diversidade nesse ambiente ter sido menor pode estar associado a diferentes espécies vegetais que ocorrem nesses ambientes, o que afeta a diversidade de insetos naquele local. De acordo com Andow (1991), a teoria ecológica sugere fatores importantes que levam a uma maior riqueza de espécies em locais mais heterogêneos, tendo estes a maior diversidade de habitats e a maior densidade de inimigos naturais, levando ao aumento de controle de populações de organismos dominantes. 
Embora apresente maior quantidade de indivíduos, o plantio consorciado teve menor diversidade que a floresta nativa. Tal fato ocorre porque a distribuição dos indivíduos em uma floresta natural é mais uniforme, em razão do maior equilíbrio ambiental, o que faz com que ocorra menor quantidade de insetos, mas com melhor distribuição dos indivíduos, propiciando maior diversidade. Um plantio, por sua vez, apresenta grande disponibilidade de alimentos, mas apenas para determinados grupos de insetos que se sobressaiam por estarem adaptados àquelas espécies plantadas, o que resulta em aumento populacional de indivíduos, embora com menor diversidade.

A população de Semiothisa sp.3 foi dominante em todos os ambientes estudados e teve um pico populacional no mês de setembro, apresentando um declínio populacional, conforme a intensidade das chuvas. É possível observar em novembro um leve aumento da população, que corresponde ao período chuvoso (Figura 2). As espécies Synchlora gerularia e Synchlora sp.2 ocorreram em todos os ambientes como dominantes, sendo registrados picos populacionais nos meses de setembro e novembro, respectivamente.

Segundo Odum (1988), as flutuações anuais podem ser controladas por fatores extrínsecos, como temperatura e precipitação pluviométrica. Silveira Neto (1972) afirma que as populações de insetos podem aumentar ou diminuir, devido a fatores favoráveis ou desfavoráveis do meio. O levantamento e a flutuação populacional de insetos são importantes para a previsão de ocorrência de possíveis surtos de pragas, pois pode-se perceber as alterações na quantidade de indivíduos ou surtos, que ocorrem ao longo do ano (Samways, 1995).

Pode ser observado na análise de agrupamento, em relação à similaridade da fauna de Geometridae coletados nos ambientes, três grupos principais, em que capoeira e mata ripária foram ambientes semelhantes, houve uma grande distância entre o plantio de teca, sendo esse mais similar à floresta nativa, enquanto o plantio consorciado demonstrou ser o mais dissimilar de todos os ambientes (Figura 3).

Tabela 4. Análise faunística das espécies de geometrídeos coletados com armadilha luminosa em área de mata ripária (ambiente 3) e em área de capoeira (ambiente 4) na Fazenda São Nicolau, em Cotriguaçu, MT, 2007 - 2008.

\begin{tabular}{|c|c|c|c|c|c|c|c|c|c|c|}
\hline \multirow{2}{*}{ Espécie } & \multicolumn{5}{|c|}{ Ambiente 3} & \multicolumn{5}{|c|}{ Ambiente 4} \\
\hline & $\mathbf{D}^{1}$ & $\mathbf{D}^{2}$ & $\mathbf{A}$ & $\mathbf{F}$ & $\mathbf{C}$ & $\mathbf{D}^{1}$ & $\mathbf{D}^{2}$ & $\mathbf{A}$ & $\mathbf{F}$ & $\mathbf{C}$ \\
\hline Epimecis puellaria & - & - & - & - & - & nd & nd & $\mathrm{c}$ & $\mathrm{f}$ & $\mathrm{z}$ \\
\hline Epimecis sp.1 & nd & nd & $\mathrm{d}$ & $\mathrm{pf}$ & $\mathrm{z}$ & nd & $\mathrm{d}$ & $\mathrm{c}$ & $\mathrm{f}$ & $\mathrm{z}$ \\
\hline Epimecis sp.2 & nd & nd & $\mathrm{c}$ & $\mathrm{f}$ & $\mathrm{y}$ & - & - & - & - & - \\
\hline Leuciris minutepunctaria & - & - & - & - & - & nd & nd & $\mathrm{c}$ & $\mathrm{f}$ & $\mathrm{z}$ \\
\hline Oospila sp. & - & - & - & - & - & nd & nd & $\mathrm{c}$ & $\mathrm{f}$ & $\mathrm{z}$ \\
\hline Oxydia vesulia & nd & nd & $\mathrm{d}$ & $\mathrm{pf}$ & $\mathrm{z}$ & - & - & - & - & - \\
\hline Oxydia sp.1 & nd & nd & $\mathrm{d}$ & $\mathrm{pf}$ & $\mathrm{z}$ & nd & nd & $\mathrm{c}$ & $\mathrm{f}$ & $\mathrm{z}$ \\
\hline Pero Amanda & nd & nd & $\mathrm{d}$ & $\mathrm{pf}$ & $\mathrm{z}$ & nd & nd & $\mathrm{c}$ & $\mathrm{f}$ & $\mathrm{z}$ \\
\hline Pero sp.5 & nd & nd & $\mathrm{d}$ & $\mathrm{pf}$ & $\mathrm{z}$ & - & - & - & - & - \\
\hline Phrudocentra sp. & - & - & - & - & - & nd & $\mathrm{d}$ & $\mathrm{c}$ & $\mathrm{f}$ & $\mathrm{y}$ \\
\hline Semiothisa sp.2 & nd & $\mathrm{d}$ & $\mathrm{c}$ & $\mathrm{f}$ & $\mathrm{z}$ & - & - & - & - & - \\
\hline Semiothisa sp.3 & $\mathrm{sd}$ & $\mathrm{sd}$ & $\mathrm{sa}$ & sf & $\mathrm{y}$ & $\mathrm{sd}$ & $\mathrm{sd}$ & $\mathrm{sa}$ & sf & $\mathrm{w}$ \\
\hline Synchlora gerularia & $\mathrm{d}$ & $\mathrm{d}$ & $\mathrm{ma}$ & $\mathrm{mf}$ & $\mathrm{y}$ & $\mathrm{d}$ & $\mathrm{d}$ & $\mathrm{ma}$ & $\mathrm{mf}$ & $\mathrm{y}$ \\
\hline Synchlora sp.1 & - & - & - & - & - & nd & nd & $\mathrm{c}$ & $\mathrm{f}$ & $\mathrm{z}$ \\
\hline Synchlora sp.2 & $\mathrm{d}$ & $\mathrm{d}$ & $\mathrm{ma}$ & $\mathrm{mf}$ & $\mathrm{y}$ & nd & $\mathrm{d}$ & $\mathrm{c}$ & $\mathrm{f}$ & $\mathrm{y}$ \\
\hline Thyrinteina schadeana & nd & $\mathrm{d}$ & $\mathrm{c}$ & $\mathrm{f}$ & $\mathrm{z}$ & nd & nd & $\mathrm{c}$ & $\mathrm{f}$ & $\mathrm{Z}$ \\
\hline Diversidade & & & 2,44 & & & & & 79 & & \\
\hline
\end{tabular}

D1 = dominância pelo método de Larroca e Mielke (1975); D2 = dominância pelo metodo de Sakagami e Larroca (1967) - (sd) super dominante, (d) dominante, (nd) não dominante; $\mathrm{A}$ = abundância - (sa) super abundante, (ma) muito abundante, (a) abundante, (c) comum, (d) dispersa, (r) rara; $\mathrm{F}=$ frequência - (mf) muito frequente, (f) frequente, (pf) pouco frequente; $\mathrm{C}=$ constância - (w) constante, (y) acessória, (z) acidental. 
Tabela 5. Análise faunística das espécies de geometrídeos coletados com armadilha luminosa em área de floresta nativa (ambiente 5), na fazenda São Nicolau, Cotriguaçu, MT, 2007 - 2008.

\begin{tabular}{|c|c|c|c|c|c|}
\hline \multirow[b]{2}{*}{ Espécie } & \multicolumn{5}{|c|}{ Ambiente 5} \\
\hline & $D^{1}$ & $\mathbf{D}^{2}$ & $\mathbf{A}$ & $\mathbf{F}$ & $\mathbf{C}$ \\
\hline Epimecis puellaria & nd & nd & $\mathrm{c}$ & $\mathrm{f}$ & z \\
\hline Oospila sp. & $\mathrm{d}$ & $\mathrm{d}$ & $\mathrm{c}$ & $\mathrm{f}$ & z \\
\hline Oxydia agliata & nd & nd & $\mathrm{c}$ & $\mathrm{f}$ & z \\
\hline Oxydia sp.1 & nd & nd & $\mathrm{d}$ & $\mathrm{pf}$ & $\mathrm{z}$ \\
\hline Pero amanda & nd & nd & $\mathrm{c}$ & $\mathrm{f}$ & $\mathrm{y}$ \\
\hline Pero sp.1 & nd & nd & d & $\mathrm{pf}$ & $\mathrm{z}$ \\
\hline Pero sp. 2 & nd & nd & $\mathrm{d}$ & $\mathrm{pf}$ & $\mathrm{z}$ \\
\hline Pero sp.4 & nd & nd & $\mathrm{c}$ & $\mathrm{f}$ & $\mathrm{z}$ \\
\hline Phrudocentra sp. & nd & nd & $\mathrm{c}$ & $\mathrm{f}$ & $\mathrm{y}$ \\
\hline Semiothisa sp.1 & $\mathrm{d}$ & $\mathrm{d}$ & $\mathrm{c}$ & $\mathrm{f}$ & $\mathrm{z}$ \\
\hline Semiothisa sp.2 & nd & nd & $\mathrm{c}$ & $\mathrm{f}$ & $\mathrm{y}$ \\
\hline Semiothisa sp.3 & d & d & $\mathrm{ma}$ & $\mathrm{mf}$ & w \\
\hline Sphacelodes vulneraria & nd & nd & $\mathrm{d}$ & $\mathrm{pf}$ & $\mathrm{z}$ \\
\hline Synchlora gerularia & d & $\mathrm{d}$ & $\mathrm{c}$ & $\mathrm{f}$ & $\mathrm{y}$ \\
\hline Synchlora sp.1 & nd & nd & $\mathrm{c}$ & $\mathrm{f}$ & $\mathrm{z}$ \\
\hline Synchlora sp.2 & d & $\mathrm{d}$ & $\mathrm{ma}$ & $\mathrm{mf}$ & $\mathrm{w}$ \\
\hline Synchlora sp.3 & nd & nd & d & $\mathrm{pf}$ & $\mathrm{z}$ \\
\hline Synchlora sp.4 & nd & nd & $\mathrm{d}$ & $\mathrm{pf}$ & $\mathrm{z}$ \\
\hline Diversidade & & & 3,75 & & \\
\hline
\end{tabular}

D1 = dominância pelo método de Larroca e Mielke (1975); D2 = dominância pelo metodo de Sakagami e Larroca (1967) - (sd) super dominante, (d) dominante, (nd) não dominante; $\mathrm{A}$ = abundância - (sa) super abundante, (ma) muito abundante, (a) abundante, (c) comum, (d) dispersa, (r) rara; $\mathrm{F}=$ frequência $-(\mathrm{mf})$ muito frequente, $(\mathrm{f})$ frequente, $(\mathrm{pf})$ pouco frequente; $\mathrm{C}=$ constância $-(\mathrm{w})$ constante, $(\mathrm{y})$ acessória, $(\mathrm{z})$ Acidental.

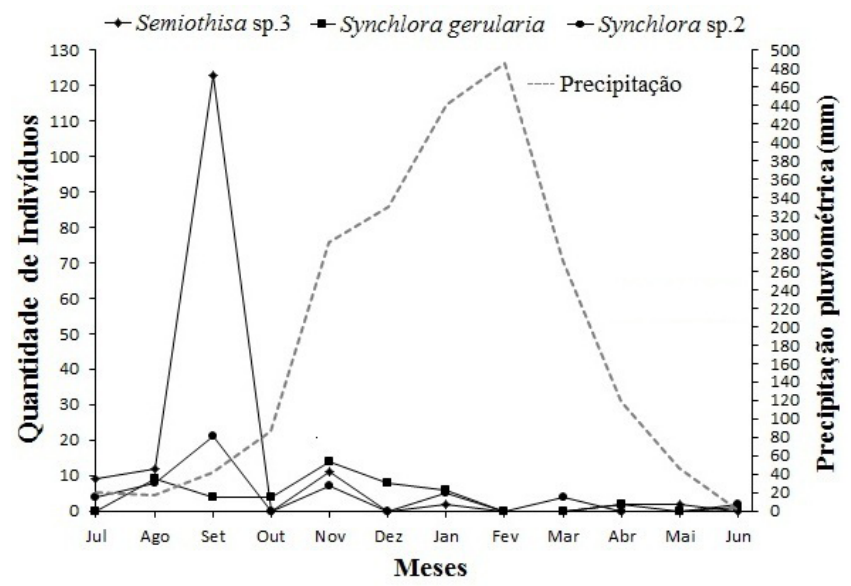

Figura 2. Flutuação populacional das espécies de geometrídeos Semiothisa sp.3, Synchlora gerularia e Synchlora sp.2 e precipitação pluviométrica (mm) na Fazenda São Nicolau, Cotriguaçu, MT, 2007-2008.

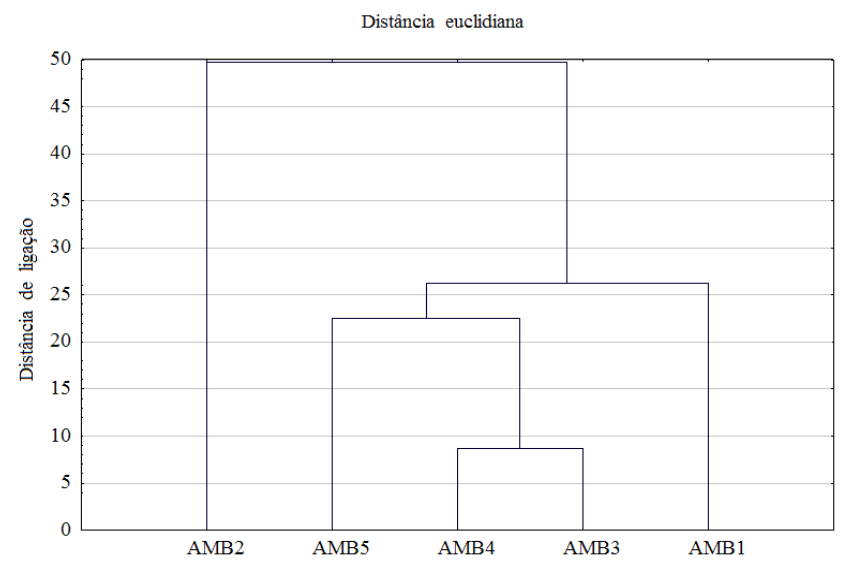

Figura 3. Dendrograma do estudo de similaridade entre os ambientes: plantio consorciado (AMB2), floresta nativa (AMB5), mata ripária (AMB4), capoeira (AMB3) e plantio de Tectona grandis (AMB1) da Fazenda São Nicolau, Cotriguaçu, MT, 2007 - 2008.

Tal fato ocorreu devido à floresta de teca apresentar características semelhantes à floresta nativa, em relação a distribuição e ocorrência dos insetos encontrados nos ambiente.

A capoeira e a mata ripária apresentam um ambiente estável ecologicamente, pois são ambientes compostos por vegetação nativa e apresentam equilíbrio ambiental, propiciando uma maior diversificação e apresentando maior similaridade com a floresta nativa em relação aos outros ambientes.

Foram observadas diferenças estatísticas significativas entre os fatores estudados (espécie x ambiente), onde algumas espécies apresentaram maior preferência por algum determinado ambiente, como a espécie Synchlora sp.2, que ocorreu em todos os ambientes, porém sua preferência foi significativamente maior no ambiente de floresta nativa e plantio consorciado (Tabela 6). De acordo com Paine \& Stephen (1987), a predominância de uma determinada espécie em um povoamento homogêneo e associação deste inseto à espécie vegetal é expressa por sua adaptabilidade a fatores relacionados à espécie vegetal hospedeira e à característica do talhão, como idade, diâmetro e crescimento radial.

A implantação de povoamentos florestais pode levar os lepidópteros desfolhadores, que antes se alimentavam exclusivamente de plantas nativas, a atacar também os povoamentos implantados (Anjos et al. 1986). Gallo et al. (2002) relatam a importância dos geometrídeos como pragas florestais de grande potencial de dano. Entre os 
inúmeros relatos de ataques e surtos de lepidópteros desfolhadores, Martins et al. (1984) descreveram a ocorrência de um complexo de lagartas da família Geometridae, atuando em conjunto: Melanolophia apicalis (Warren, 1900), Glena bipennaria bipennaria (Guenée, 1857) e Pherotesia confusata (Walker, 1862), na qual causaram danos econômicos em Pinus patilla, Telêmaco Borba, PR.

Tabela 6. Quantidade média de indivíduos de geometrídeos coletados nos diferentes ambientes estudados na Fazenda Mutuca, em Cotriguaçu, MT, 2007-2008.

\begin{tabular}{|c|c|c|c|c|c|c|c|c|}
\hline \multirow{2}{*}{ Espécies } & \multicolumn{8}{|c|}{ Ambientes ${ }^{1}$} \\
\hline & 1 & & 2 & & 3 & 4 & 5 & \\
\hline Epimecis puellaria & - & & - & & - & $0,87 \mathrm{cA}$ & 1,05 & $\mathrm{dA}$ \\
\hline Epimecis sp.1 & - & & - & & 0,87 eA & $1,17 \mathrm{bA}$ & - & \\
\hline Epimecis sp.2 & - & & - & & $1,17 \mathrm{~d}$ & - & - & \\
\hline Leuciris minutepunctaria & - & & - & & - & $0,87 \mathrm{c}$ & - & \\
\hline Oospila sp. & 1,17 & $\mathrm{cB}$ & 0,87 & $\mathrm{eB}$ & - & $1,05 \mathrm{bB}$ & 1,64 & $\mathrm{cA}$ \\
\hline Oxydia agicata & - & & - & & - & - & 1,17 & $\mathrm{c}$ \\
\hline Oxydia vesulia & - & & - & & $0,87 \mathrm{e}$ & - & - & \\
\hline Oxydia sp. & - & & - & & $0,87 \mathrm{eA}$ & $0,87 \mathrm{cA}$ & 0,87 & $\mathrm{dA}$ \\
\hline Pero amanda & 0,87 & $\mathrm{c}$ & - & & 0,87 eA & $0,87 \mathrm{cA}$ & 1,22 & $\mathrm{cA}$ \\
\hline Pero sp.1 & 0,87 & $\mathrm{cA}$ & 0,99 & $\mathrm{eA}$ & - & - & 0,87 & $\mathrm{dA}$ \\
\hline Pero sp.2 & - & & 0,87 & $\mathrm{eA}$ & - & - & 0,87 & $\mathrm{dA}$ \\
\hline Pero sp.3 & 0,87 & $\mathrm{c}$ & - & & - & - & - & \\
\hline Pero sp.4 & 1,26 & $\mathrm{cA}$ & 1,55 & $\mathrm{dA}$ & - & - & 0,99 & $\mathrm{~dB}$ \\
\hline Pero sp.5 & - & & - & & $0,87 \mathrm{e}$ & - & - & \\
\hline Phrudocentra sp. & 0,87 & $\mathrm{cB}$ & 1,34 & $\mathrm{dA}$ & - & $1,17 \mathrm{bA}$ & 1,46 & $\mathrm{cA}$ \\
\hline Semiothisa sp.1 & 0,99 & $\mathrm{cB}$ & 0,87 & $\mathrm{eB}$ & - & - & 1,55 & $\mathrm{cA}$ \\
\hline Semiothisa sp.2 & 2,01 & $\mathrm{bB}$ & 2,71 & $\mathrm{bA}$ & $1,26 \mathrm{dC}$ & - & 1,44 & $\mathrm{cC}$ \\
\hline Semiothisa sp. 3 & 3,26 & $\mathrm{aB}$ & 4,36 & $\mathrm{aA}$ & $2,95 \mathrm{aB}$ & $2,58 \mathrm{aC}$ & 3,16 & $\mathrm{aB}$ \\
\hline Sphacelodes vulneraria & 0,87 & $\mathrm{cA}$ & 0,87 & $\mathrm{eA}$ & - & - & 0,87 & $\mathrm{dA}$ \\
\hline Synchlora gerularia & 2,01 & $\mathrm{bA}$ & 1,55 & $\mathrm{~dB}$ & $2,11 \mathrm{bA}$ & 2,14 aA & 1,71 & $\mathrm{cB}$ \\
\hline Synchlora sp.1 & - & & 0,87 & $\mathrm{eA}$ & - & $0,87 \mathrm{cA}$ & 1,05 & $\mathrm{dA}$ \\
\hline Synchlora sp.2 & 0,87 & $\mathrm{cC}$ & 2,14 & $\mathrm{cA}$ & $1,64 \mathrm{cB}$ & $1,46 \mathrm{bB}$ & 2,28 & bA \\
\hline Synchlora sp.3 & - & & - & & - & - & 0,87 & d \\
\hline Synchlora sp.4 & - & & - & & - & - & 0,87 & d \\
\hline Thyrinteina schadeana & - & & 0,87 & $\mathrm{eB}$ & $1,34 \mathrm{dA}$ & $0,87 \mathrm{cB}$ & - & \\
\hline $\mathrm{F}$ & & & & & 3,15 & & & \\
\hline $\mathrm{CV}(\%)$ & & & & & 27,04 & & & \\
\hline
\end{tabular}

${ }^{1}$ Dados transformados em $\sqrt{X+0,5}$. Médias seguidas de mesma letra minúscula nas colunas e maiúscula nas linhas, não diferem entre si ao nível de $5 \%$ de probabilidade pelo teste Scott-Knott. Ambientes: (1) plantio de teca; (2) plantio consorciado; (3) capoeira; (4) mata ripária; e (5) floresta nativa. 


\section{Conclusões}

A floresta nativa apresenta melhor distribuição dos indivíduos da fauna de geometrídeos, apresentando maior diversidade. $\mathrm{O}$ aumento das chuvas faz com que ocorra uma diminuição da quantidade de indivíduos de geometrídeos nos ambientes. As espécies Semiothisa sp.3, Synchlora gerularia e Synchlora sp.2 estão adaptadas em todos os ambientes estudados.

\section{Agradecimentos}

À Organización Nacionale Du Fôret - ONF Brasil Ltda., por ter cedido o local para o estudo, ao Professor Dr. Sinval Silveira Neto, da Escola Superior de Agricultura "Luiz de Queiroz" (ESALQ - USP), pela identificação dos Lepidópteros, e ao técnico do laboratório de proteção florestal/FENF-UFMT, Manoel Lauro da Silva, pelo auxílio no desenvolvimento da pesquisa.

\section{Referências}

ANDOW, D. A. Vegetational diversity and arthropod population response. Annual Review of Entomology, Palo Alto, v. 35, p. 561-586, 1991.

ANJOS, N.; SANTOS, G. P.; ZANUNCIO, J. C. Pragas do eucalipto e seu controle. Informe Agropecuário, Belo Horizonte. v. 12, p. 50-58, 1986.

BROWN JUNIOR, K. S.; FREITAS, A. V. L. Atlantic forest butterflies: indicators for landscape conservation. Biotropica, Lawrence, v. 32, n. 4b, p. 934-956, 2000.

DORVAL, A.; PERES FILHO, O.; MARQUES, E. N. Levantamento de Scolytidae (Coleoptera) em plantações de Eucalyptus spp. em Cuiabá, estado de Mato Grosso. Ciência Florestal, Santa Maria, RS, v. 14, n. 1, p. 47-58, 2004.

FERREIRA, D. F. SISVAR: um programa para análises e ensino de estatística. Revista Symposium, Lavras, v. 6, p. 36-41, 2008.

FERREIRA, P. S. F.; MARTINS, D. S. Contribuição ao método de captura de insetos por meio de armadilha luminosa, para obtenção de exemplares sem danos morfológicos. Revista Ceres, Viçosa, MG, v. 29 , p. $538-543,1982$.

FONSECA, N. G.; KUMAGAII, A. F.; MIELKE, O. H. H. Lepidópteros visitantes florais de Stachytarpheta cayennensis (Rich.) Vahl (Verbenaceae) em remanescente de Mata Atlântica, Minas Gerais, Brasil. Revista Brasileira de Entomologia, São Paulo, v. 50, n. 3, p. 399-405, 2006.

GALLO, D.; NAKANO, O.; SILVEIRA NETO, S.; CARVALHO, R. P. L.; BAPTISTA, G. C.; BERTI FILHO, E.; PARRA, J. R. P.; ZUCCHI, R. A.; ALVES, S. B.; VENDRAMIM, J. D.; MARCHINI, L. C.; LOPES, J. R. S.; OMOTO, C. Entomologia Agrícola. Piracicaba: FEALQ, 2002. 920 p.
LAROCA, S.; MIELKE, O. H. H. Ensaios sobre ecologia de comunidades em Sphingidae da Serra do Mar. Paraná, Br. (Lepidoptera). Revista Brasileira de Biologia, Rio de Janeiro, v. 35, n. 1, p. 1-19, 1975.

LOURENÇÃO, A. L.; REZENDE, J. A. M.; MASCARENHAS, H. A. A.; FERREIRA FILHO, A. W. P. Ocorrência de Semiothisa abydata (guenée) (Lepidoptera: Geometridae) em soja no estado de São Paulo. Bragantia, Campinas, v. 39, n. 18, 1980.

MARGALEF, R. Ecologia. Barcelona: Omega, 1951. 951 p.

MARGALEF, R. Homage to Evelyn Hutchinson, or why is there an upper limit to diversity. Transactions of the Connecticut Academy of Arts and Sciences, New Haven, v. 44, p. 211-235, 1972.

MARGULES, C. R.; PRESSY, R. L. Systematic conservation planning. Nature, London, v. 405, p. 243-253, 2000.

MARTINS, A. J.; MACEDO, J. H. C.; SANTOS, H. R.; CORDEIRO, L. Melanolophia apicalis (Warren, 1900) em Pinus pátula no Paraná. Revista Floresta, Curitiba, v. 15, n. 1, p. 81-85, 1984.

MORAES, R. C. B.; HADDAD, M. L.; SILVEIRA NETO, S.; REYES, A. E. L. Software para análise faunística. In: SIMPÓSIO DE CONTROLE BIOLÓGICO, 8., 2003, São Pedro, SP. Resumos. Piracicaba: SEB, 2003. 195 p.

MOTTA, P. C. Butterflies from the Uberlândia region, central Brazil: species list and biological comments. Brazilian Journal of Biology, São Carlos, v. 62, n. 1, p. 151-163, 2002.

NAKANO, O.; LEITE,C. A. Armadilhas para insetos: pragas agrícolas e domésticas. Piracicaba: FEALQ, 2000. 76 p.

ODUM, E. Ecologia. Rio de Janeiro: Guanabara Koogan, 1988. $434 \mathrm{p}$.

PAINE, T. D.; STEPHEN, F. M. The relationship of tree height and crown class to the induced plant defenses of loblolly pine. Canadian Journal of Botany, Guelph, v. 65, n. 2, p. 2090-2092, 1987.

PERES FILHO, O.; DORVAL, A.; SCHMIDIT, V.; BERTI FILHO, E.; MOURA, R. G. Biologia de Thyrinteina arnobia em folhas de Eucalyptus camaldulensis, Corymbia citriodora, Urograndis, Psidium guajava e Vernonia condensata. Revista de Agricultura Piracicaba, v. 85, p. 1-14, 2010.

PHILLIPS, T. W. Responses of Hylastes salebrosus to turpentine, ethanol, and pheromones of Dendroctonus (Coleoptera: Scolytidae). The Florida Entomologist, Gainesville, v. 73, n. 2, p. 286-292, 1990.

PRATISSOLI, D.; FONAZIER, M. J. Ocorrência de Trichogramma acacioi Brun, Moraes \& Soares (Hymenoptera: Trichogrammatidae) em ovos de Nipteria panacea Thierry-Mieg (Lepidoptera: Geometridae), um geometrídeo desfolhador do abacateiro. Anais da Sociedade Entomológica do Brasil, Jaboticabal, v. 28, n. 2, p. 347-349, 1999.

PROJETO RADAMBRASIL. Folha SC 21 Juruena. Rio de Janeiro: Departamento de Produção Mineral, 1982. (Levantamento de Recursos Naturais, 20).

SAKAGAMI, S. F.; LAROCA, S. Observations on the bionomics of some neotropical Xylocopini bees, with some comparative biofaunistic notes (Hymenoptera, Anthophoridae). Journal of the Faculty of Science, Hokkaido, v. 18, p. 57-127, 1967. 
SAMWAYS, M. J. Insect conservation biology. London: Chapman, $1995.358 \mathrm{p}$.

SANTOS, G. P.; ZANUNCIO, T. V.; VINHA, E.; ZANUNCIO, J. C. Influência de faixas de vegetação nativa em povoamentos de Eucalyptus cloeziana sobre população de Oxydia vesulia (Lepidoptera: Geometridae). Revista Árvore, Viçosa, MG, v. 26, n. 4, p. 499-504, 2002.

SILVEIRA NETO, S. Levantamento de insetos e flutuação da população de pragas da Ordem Lepidóptera, com o uso de armadilhas luminosas, em diversas regiões do Estado de São Paulo. 1972. 182 f. Tese (Livre-Docência) - Escola Superior de Agricultura "Luiz de Queiroz", Universidade de São Paulo, Piracicaba.
SILVEIRA NETO, S.; NAKANO, O.; BARBIN, D.; VILLA NOVA, N. A. Manual de ecologia dos insetos. São Paulo: Agronômica Ceres, 1976. 419 p.

SILVEIRA NETO, S.; SILVEIRA, A. C. Armadilha luminosa modelo "Luiz de Queiroz". O Solo. Piracicaba, v. 61, n. 2, p. 19-21, 1969.

WARD, J. H. Hierarchical grouping to optimize an objective function. Journal of the American Statistical Association. Alexandria, US, v. 58, p. $236,1963$.

YAMAMOTO, S.; SOTA, T. Phylogeny of the Geometridae and the evolution of winter moths inferred from a simultaneous analysis of mitochondrial and nuclear genes. Molecular Phylogenetics and Evolution, v. 44, p. 711-723, 2007. 